\title{
Adaptable Source-Grid Planning for High Penetration of Renewable Energy Integrated System
}

\author{
Ming Tang ${ }^{1}$, Jian Wang ${ }^{2, *}$ and Xiaohua Wang ${ }^{2}$ \\ 1 College of Electrical Engineering, Zhejiang University, Hangzhou 310027, China; tangmingtzju@zju.edu.cn \\ 2 Tsinghua Sichuan Energy Internet Research Institute, Chengdu 610213, China; \\ wangxiaohuawxh@tsinghua-eiri.org \\ * Correspondence: wjqhscnyy@163.com
}

Received: 4 June 2020; Accepted: 26 June 2020; Published: 28 June 2020

check for updates

\begin{abstract}
To adapt to the growing scale of renewable energy and improve the consume ability of the power system, it is necessary to design a highly adaptable planning scheme for high penetration of the renewable energy integrated system. Thus, this paper firstly gives the conception of system adaptability and designs an adaptability index system, which considers the supply and demand balance, operation state, and network structure of the high penetrated renewable energy integrated system. It can help to comprehensively evaluate the system ability towards uncertain shocks. Then, a two-stage source-grid coordinative expansion planning model is presented. The adaptability indexes of supply and demand balance are used as objection of the source planning stage, the adaptability indexed of the operation state and network structure are used to guide the grid planning stage. The model is further solved based on the coordination between the source and grid planning stage. Finally, the case study verifies that the obtained optimal plan has good adaptability to the impact of renewable energy on the power supply capacity and security operation.
\end{abstract}

Keywords: high penetration; renewable energy; adaptability planning; source-grid coordination

\section{Introduction}

\subsection{Motivation}

The pursuit of a sustainable low-carbon society has made renewable energy, represented by wind power and photovoltaics, popularized and applied on a large scale. With the rapid development of related technologies and the breakthrough of key issues, the penetration of renewable energy increases each year [1]. The newly installed capacity of renewable energy units in the world reached $181 \mathrm{GW}$ by 2018 , in which wind and photovoltaic power accounted for $83 \%$, while the renewable energy unit supplied more than $26 \%$ of load demand [2]. The obvious influence of natural resources and seasonal climate cause the renewable energy unit to have strong volatility and randomness [3]. Variable renewable energy sources, such as solar photovoltaic and wind power, not only change the patterns of electricity utilization on the demand side, but also bring challenges for the balancing and security of power supply on the source side [4]. Considering the scale effect, the uncertain characteristics of multi-spatiotemporal differential distribution of renewable energy are coupled in the operation features of the power system. That seriously impacts the safety and stability of the power system and brings great challenges to the planning and operation. The limitation of network structure and shortage of flexibility resources will further restrict the adaptability of the power system for renewable energy integration. Thus, a source-grid coordinative planning scheme adaptable for the high penetration system is crucial to improve the system adaptability to renewable energy [5]. 


\subsection{Previous Work}

To reduce the impact of renewable energy uncertainties on the power system and maximize its advantages of cleanliness, high efficiency, and environment friendly, existing research analyzes the adaptability of high penetration of the renewable energy integrated system [6]. Flexibility is an important theory that is currently widely studied and used to solve the uncertain impact of high penetration of renewable energy. This theory mainly analyzes the ability of flexibility resources in the system to copy with the demand under uncertain operating environments [7]. The characteristics of flexibility such as directionality, time scale, and the key points of flexible assessment were summarized in [8]. A quantitative assessment index of flexibility based on interval evaluation was established in [9]. Based on the flexibility assessment, the flexibility balance mechanism was explored in [10] and various types of flexibility coordination planning models were established. Although flexibility assessment is an important part of the scope of adaptability analysis, it pays more attention to the adaptability of supply and demand balance and lacks consideration of the state of the power system. The impact of high penetration of renewable energy is not only reflected in the supply and demand balance, but also needs to consider the safety, stability, economic efficiency of system operation, and also the affordability of the network structure. The system adaptability characterizes the ability to achieve optimal development in the mutual influence and coordination between itself and external factors, it can more fully reflect the consumption ability for renewable energy integration [11]. Hence, considering the impact of high penetration of renewable energy on other aspects of the power system, [12] analyzed the adaptability of the power system to integrated photovoltaic power plants from the perspective of voltage stability and power flow safety and proposed adaptive expansion measures for reactive power improvement. The influence of the centralized wind farm on the evolution of the self-organized state of the power system is discussed in [13] based on the entropy theory. [14] further considered the probabilistic operating characteristics of high penetration of the renewable energy integrated system and proposed an adaptive evaluation index system from the prospects of safety, economy, and stability. As far as current research is concerned, the system adaptability to the renewable energy integrated system mainly focuses on the adaptability of existing power systems, which belongs to the category of post evaluation and rarely works on the relevant application of adaptability evaluation to the source and grid expansion planning of the power system [15].

\subsection{Current Contribution}

Based on the above, this paper proposed an adaptable source-grid coordinated expansion plan for high penetration of the renewable energy integrated system with the help of the adaptability evaluation. Firstly, an adaptability index system for high penetration of the renewable energy integrated system towards source-grid expansion planning is presented in this paper, which covers three aspects: adaptability of the supply and demand balance, adaptability of the operating state, and adaptability of the network structure. Among them, the state evaluation model for supply and demand balance is established considering the directivity of supply and demand regulation, diversity of time scale, and complexity of network constraint, then the adaptability index comprising both the insufficient rate and insufficiency of supply and demand is presented. Quantifying the fluctuation and variable operation state of high penetration of the renewable energy integrated system, the weighted entropy index of the expected line load rate that takes into account the safety and economic operation and the weighted entropy index of the line power fluctuation rate to evaluate the stability operation are proposed, then a comprehensive index is designed based on joint weighted entropy to evaluate the rationality of the system operation state. Quantifying the electrical characteristics of the network structure, the weighted entropy index that considers the safety of bus and branch is proposed, then a joint weighted entropy index of the network structure is established to reflect the robustness of the overall structure. Furthermore, based on the proposed index system and accounting for the system safety constraints, power flow constraints, equipment operation constraints, and renewable energy output volatility, an adaptable source-grid coordinative two-stage expansion planning model is 
proposed, which combines the source planning and the gird planning. While preserving the power balance and regulation capabilities, the multiple attributes of the operation state and network structure are optimized. The matching and adaptability between the source planning scheme and grid planning scheme are enhanced, improving consumption ability of the system to high penetration of renewable energy. The simulation and analysis carried out in the Garver- 18 bus test system verify the practicability and effectiveness of the proposed method.

\subsection{Structure}

The rest of this paper is organized as follows. Section 2 presents the system adaptability index system. Section 3 introduces the adaptive source-grid coordinative expansion planning model and its solution method. The case study is illustrated in Sections 4 and 5 concludes this paper.

\section{Evaluation of the System Adaptability to High Penetration of Renewable Energy}

Renewable energy is obviously restricted by natural resources and its essential feature is strong uncertainty with multi-temporal distribution. Its large-scale integration will have a strong impact on all aspects of the power system, as shown in Figure 1. It can be seen that under a new background, fast and sufficient regulation resources are the key to ensuring the balance between supply and demand [16].This paper defines the adaptability of the high penetration of the renewable energy integrated system as: under the uncertain operating environment of high penetration of renewable energy integration, power system calls for fast regulation resources to respond to the changes in demand and relies on a robust topology to resist uncertain shocks. On the basis of the balance between the supply and demand under various time scales, the ability to maintain the safe, efficient, and stable operation state of the power system is always maintained. Combined with the above definition, we can see that the evaluation perspective of the adaptability of high penetration of the renewable energy integrated system includes three parts: multi-time scale power supply and demand balance, high-quality and reliable level of operation state, and robustness of network topology.

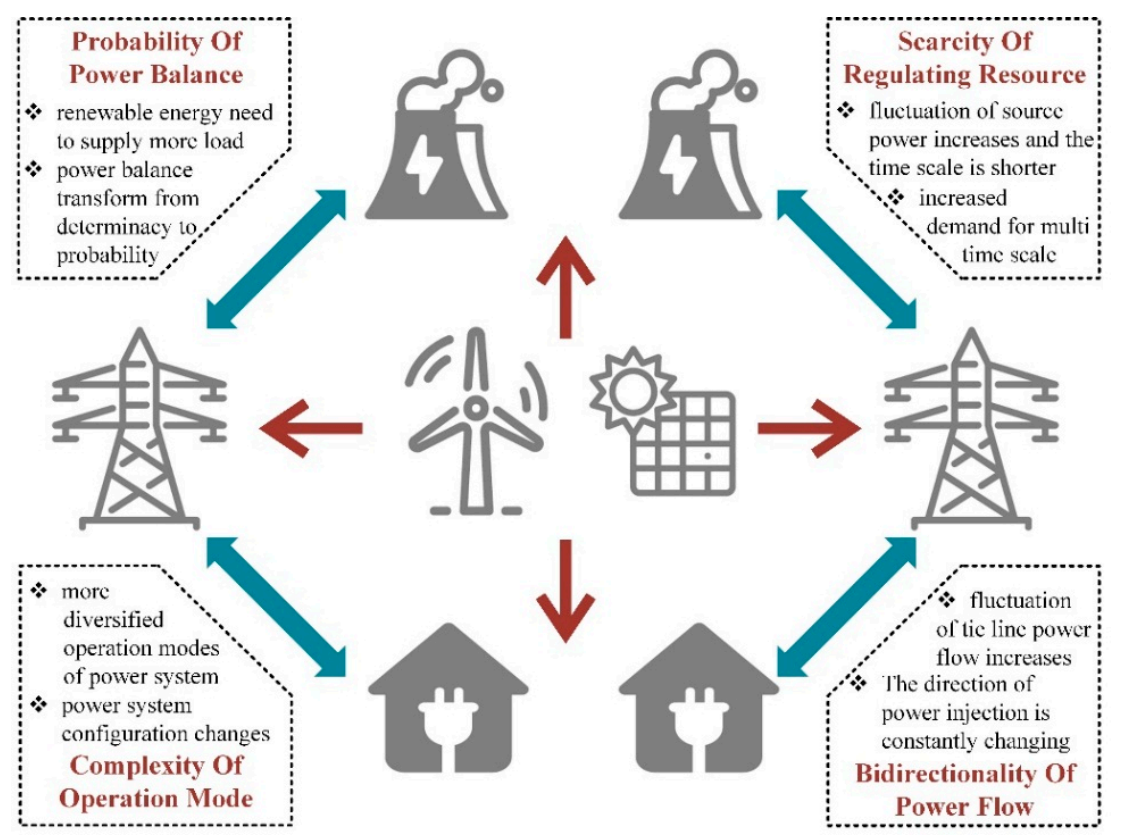

Figure 1. Typical characteristics of high penetration of the renewable energy integrated system.

\subsection{Adaptability Index System}

The adaptability index system should take into account the typical characteristics of high penetration of renewable energy, which is suitable for the new background with multi-spatiotemporal 
uncertain characteristics and then effectively characterizes the adaptability to the uncertain impact of renewable energy from multiple perspectives [17]. At the same time, the design of the index system should be oriented to be a guidance criterion for source-grid planning. Here, the proposed index system for the adaptability of high penetration of the renewable energy integrated system is given in Figure 2.

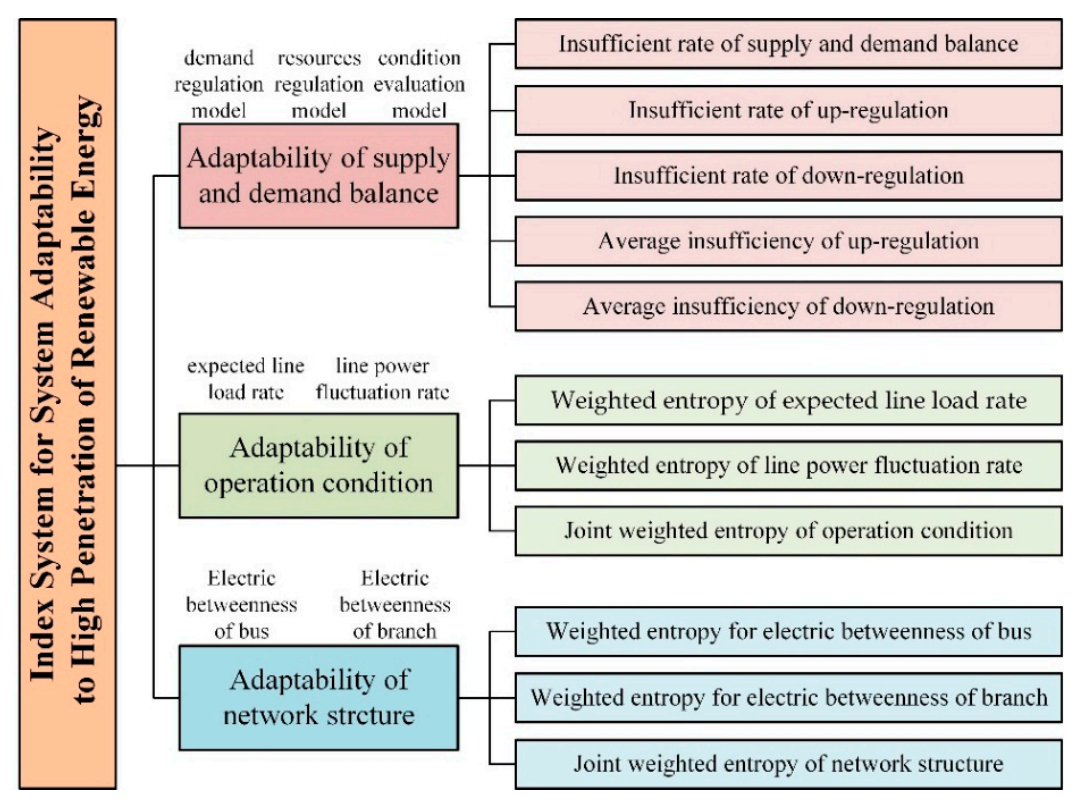

Figure 2. Evaluation index system for system adaptability to high penetration of renewable energy.

\subsection{Adaptability of Supply and Demand Balance}

Adaptability of supply and demand balance means the ability to maintain multi-time scale balance of supply and demand, where the system responds to the regulation demand caused by the fluctuating output of renewable energy by fast regulation resources. In this section, we firstly introduce the basic characteristics, and the quantitative evaluation model of supply and demand balance considering network constraints is proposed. Then, we give the indexes in detail to evaluate the probability and average degree of supply and demand shortage.

\subsubsection{Basic Characteristics}

\section{(1) Directivity of Supply and Demand Regulation}

The regulation demand of traditional power systems mainly comes from the power shortage caused by faults. Specifically, when the power system lacks power supply, spare capacity is required to fill the power gap in time to ensure the supply and demand balance. So, the fault regulation demand has a single directionality, i.e., up-regulation. However, under the high penetration of renewable energy integration, renewable energy sources have become the main source of the power supply and will bear most of the load demand. Due to the strong fluctuation and intermittency of renewable energy, its output power will continuously change with time and the direction of change is uncertain. In this situation, the system needs to be able to quickly adjust the resources to match it, respond to changes in the renewable energy, form a complementary relationship of upward and down-regulation, and ultimately ensure reliable power supply and efficient consumption of renewable energy. Therefore, the high penetration of the renewable energy integrated system has the regulation demand coming from the renewable energy power change and its directionality has the upward and downward bidirectional characteristics. 


\section{(2) Diversity of Time Scale}

When there is a generator and transmission line failure, it will cause up-regulation demand. However, with the improvement of the safety and reliability of the power system, the probability of its occurrence is very low and the frequency of the up-regulation demand is the same as the frequency of the failure. At the same time, the response time of its regulation is usually required to be within 10-30 $\mathrm{min}$, which is a medium-long regulation time scale. However, because the output power of renewable energy varies with natural resources, the fluctuation is relatively frequent and there are extreme cases of steep drop and steep increase. Therefore, the frequency of upward and down-regulation requirements resulting from changes in the output of renewable energy is relatively higher. Similarly, the response time of the regulation resources is shorter, which belongs to the short-term regulation time scale. Comparing with the temporal characteristics of traditional reserve regulation, the regulation demand caused by renewable energy has a higher frequency and shorter time scale.

\section{(3) Complexity of Network Constraint}

The actual regulation capability of the power system is determined by the capacity of the system configuration regulation resource and network structure, the capacity of the regulation resource configuration determines the upper limit of the system regulation capability. In the operation of the system, due to the limitation of network transmission, the actual regulation capability of the power system is often less than the regulation capacity. Therefore, in the analysis of the power supply and demand balance relationship between source and demand, it is not possible to simply use the sum relationship to determine whether the available regulation resources can match the demand. On the one hand, the quantitative relationship can theoretically reflect whether the upper limit of regulation capability can meet demand; on the other hand, when further analyzing the balance between supply and demand, power flow constraint should be added to consider the support of the network.

\subsubsection{State Evaluation Model}

\section{(1) Demand Regulation Model}

In high penetration of the renewable energy integrated system, the fluctuation of renewable energy output power is the main reason for the regulation demand. Compared with changes in load power, the fluctuation of renewable energy power is larger and more frequent and there is no corresponding rule to follow. This paper mainly considers the regulation demand caused by the fluctuation of renewable energy, the relevant regulation demand is formulated as [18]:

$$
\left\{\begin{array}{l}
P_{\mathrm{D}}^{\text {up }}(t, \tau)=\sum_{P_{i, \mathrm{REG}} \in \Omega_{\mathrm{REG}}}\left|P_{i, \mathrm{REG}}(t+\tau)-P_{i, \mathrm{REG}}(t)\right| \quad P_{i, \mathrm{REG}}(t)>P_{i, \mathrm{REG}}(t+\tau) \\
P_{\mathrm{D}}^{\text {down }}(t, \tau)=\sum_{P_{i, \mathrm{REG}} \in \Omega_{\mathrm{REG}}}\left|P_{i, \mathrm{REG}}(t+\tau)-P_{i, \mathrm{REG}}(t)\right| \quad P_{i, \mathrm{REG}}(t)<P_{i, \mathrm{REG}}(t+\tau)
\end{array}\right.
$$

where $P_{\mathrm{D}}{ }^{\text {up }}(t, \tau)$ and $P_{\mathrm{D}}{ }^{\text {down }}(t, \tau)$ represent the up- and down-regulation demand respectively; $P_{i, \text { REG }}(t)$ and $P_{i, \operatorname{REG}}(t+\tau)$ represent the output power of the renewable energy unit $i$ at the time $t$ and $t+\tau$; $\Omega_{\mathrm{REG}}$ is the set for the renewable energy unit.

It can be seen from (1) that when the output power of renewable energy decreases at $t+\tau$, up-regulation demand will be generated. If the fast regulation resources can match this demand, the power supply reliability can be maintained continuously. Otherwise, there will be a load shedding. Similarly, when the output power of renewable energy increases at $t+\tau$, there will be a down-regulation demand. If the fast regulation of resources cannot be matched, there will be a curtailment of renewable energy. 


\section{(2) Resources Regulation Model}

The regulation resources include various types of generators with rapid regulation capabilities, such as rapid regulation units, energy storage devices, etc., and various regulation methods, such as demand-side response, rapid load shedding. However, as far as the technical level and practical application of the above-mentioned various measures are concerned, energy storage devices are expensive to build and have limited capacity and are mostly used in distribution networks and below. Demand-side responses are similar. Two types of regulation measures, such as rapid load shedding and curtailment of wind and photovoltaic, are contrary to the original intention of reliable power supply and efficient consumption of renewable energy and are often used as the last level of security control. Therefore, at the transmission grid level, large-capacity fast regulating units are currently the most important regulation resource. For such resources, its ability to regulate can be expressed as [18]:

$$
\left\{\begin{array}{l}
P_{\mathrm{S}}^{\mathrm{up}}(t, \tau)=\sum_{P_{i, \mathrm{G}} \in \Omega_{\mathrm{G}}} \min \left[R_{i, \mathrm{G}}^{\mathrm{up}} \cdot \tau, P_{i, \mathrm{G}}^{\max }-P_{i, \mathrm{G}}(t)\right] \\
P_{\mathrm{S}}^{\text {down }}(t, \tau)=\sum_{P_{i, \mathrm{G}} \in \Omega_{\mathrm{G}}} \min \left[R_{i, \mathrm{G}}^{\text {down }} \cdot \tau, P_{i, \mathrm{G}}(t)-P_{i, \mathrm{G}}^{\min }\right]
\end{array},\right.
$$

where $P_{\mathrm{S}}{ }^{\text {up }}(t, \tau)$ and $P_{\mathrm{S}}{ }^{\text {down }}(t, \tau)$ represent the up- and down-regulation capability and the subscript $S$ refers to "Supply"; $\Omega_{\mathrm{G}}$ is the set of fast regulating units; $P_{i, \mathrm{G}}(t)$ is the output power of the fast regulating units $i$ at time $t ; P_{i, \mathrm{G}} \max$ and $P_{i, \mathrm{G}}{ }^{\min }$ are the upper and lower output of the fast regulating generator units respectively; $R_{i, g}$ up and $R_{i, g}$ down respectively represent the maximum up and down ramp rate of the fast regulation units.

As can be seen from (2), that the regulation capability of the fast regulation unit is determined by the current operating state and regulation capability of the unit. Taking the up-regulation capability as an example, the actual regulation capability is the smaller of the maximum climbing regulation rate, the upper limit of the output power, and the difference between the actual output power.

(3) Condition Evaluation Model for Supply and Demand Balance

$$
\begin{gathered}
\left\{\begin{array}{l}
P_{\mathrm{M}}^{\mathrm{up}}(t, \tau)=P_{\mathrm{S}}^{\mathrm{up}}(t, \tau)-P_{\mathrm{D}}^{\mathrm{up}}(t, \tau) \\
P_{\mathrm{M}}^{\text {down }}(t, \tau)=P_{\mathrm{S}}^{\text {down }}(t, \tau)-P_{\mathrm{D}}^{\text {down }}(t, \tau)
\end{array}\right. \\
\text { s.t. }-\boldsymbol{B} \boldsymbol{\theta}(t, \tau)+\boldsymbol{P}_{\mathrm{g}}(t, \tau)+\boldsymbol{P}_{\mathrm{G}}(t, \tau)+\boldsymbol{P}_{\mathrm{REG}}(t, \tau)=\boldsymbol{P}_{\mathrm{L}}(t, \tau),
\end{gathered}
$$

where $P_{\mathrm{M}}{ }^{\text {up }}(t, \tau)$ and $P_{\mathrm{M}}{ }^{\text {down }}(t, \tau)$ represent the upper and lower regulation resource margins and the subscript $\mathrm{M}$ refers to "Margin"; $\boldsymbol{B}$ is bus admittance matrix; $\boldsymbol{\theta}, \boldsymbol{P}_{\mathrm{g}}, \boldsymbol{P}_{\mathrm{G}}, \boldsymbol{P}_{\mathrm{REG}}, \boldsymbol{P}_{\mathrm{L}}$ are voltage angel vector, the output vector of the conventional generator unit integrated bus, the output vector of the fast regulation generator unit integrated bus, the output vector of the renewable energy unit integrated bus and the power vector of the load bus.

The presented state evaluation model of the supply and demand balance consists of two parts. (3) can be used to judge whether the upper limit of system regulation resources is sufficient or insufficient, so as to indicate whether the regulation resources allocated by the system can meet the demand of regulation. (4) requires that the operation calls of each unit meet the network transmission constraints. Therefore, (3) and (4) can be used to comprehensively reflect the satisfaction relationship between the actual available regulation capacity and the demand. To sum up, when $P_{\mathrm{M}}{ }^{\mathrm{up}}(t, \tau)$ or $P_{\mathrm{M}}{ }^{\operatorname{down}}(t, \tau)$ are greater than 0 , it indicates that the system can maintain the balance of power supply and demand at this time; on the contrary, there is a lack of fast regulation in the system, which will lead to a certain degree of forced load shedding or curtailment of renewable energy [18].

\subsubsection{Evaluation Indexes}

Based on the state evaluation model for supply and demand balance, further construct the adaptability indexes of supply and demand balance, which are described below. 
(1) Insufficient Rate of Supply and Demand Balance (IRSD)

IRSD is introduced to characterize the ratio of the time when the power supply and demand balance is insufficient to the total operation time, i.e., the probability of the insufficient supply and demand balance [18]:

$$
R_{\text {ins }}=\frac{T_{\text {ins }}}{T_{\text {total }}}, T_{\text {ins }}=\sum_{t \in T_{\text {total }}} t_{P_{\mathrm{M}}(t, \tau)<0}
$$

where $T_{\text {ins }}$ and $T_{\text {total }}$ represent the time of insufficient supply and demand balance and the total running time; $t_{\mathrm{PM}<0}$ represents the moment when the supply and demand balance is insufficient.

(2) Insufficient Rate of Up-Regulation (IRUR)

IRUR is introduced to characterize the ratio of the time between insufficient supply and demand balance due to lack of up-regulation resources and the total time of up-regulation demand, i.e., the probability of the supply and demand imbalance and load shedding due to lack of up-regulation resources [18]:

$$
R_{\text {ins }}^{\text {up }}=\frac{T_{\text {ins }}^{\text {up }}}{T_{\text {total }}^{\text {up }}}, T_{\text {ins }}^{\text {up }}=\sum_{t \in T_{\text {total }}^{\text {up }}} t_{P_{\mathrm{M}}^{\text {up }}(t, \tau)<0^{\prime}}
$$

where $T_{\text {ins }}$ up and $T_{\text {total }}$ up are the time when the up-regulation resources are insufficient and the total operation time when the up-regulation demand occurs respectively, $t_{\mathrm{PM}}$ up $<0$ is the time when the up-regulation is insufficient.

\section{(3) Insufficient Rate of Down-Regulation (IRDR)}

IRDR is introduced to characterize the ratio of the time when the supply and demand balance is insufficient due to the lack of down-regulation resources and the total time when the down-regulation demand occurs, i.e., the probability of the supply and demand imbalance and renewable energy curtailment due to the lack of down-regulation resources [18]:

$$
R_{\text {ins }}^{\text {down }}=\frac{T_{\text {ins }}^{\text {down }}}{T_{\text {total }}^{\text {down }}}, T_{\text {ins }}^{\text {down }}=\sum_{t \in T_{\text {total }}^{\text {down }}} t_{P_{\mathrm{M}}^{\text {down }}(t, \tau)<0^{\prime}}
$$

where $T_{\text {ins }}$ down and $T_{\text {total }}{ }^{\text {down }}$ represent the time when the down-regulation resource is insufficient and the total operation time when the down-regulation demand occurs; $t_{\mathrm{PM}}{ }^{\text {down }}<0$ represents the time when the down-regulation is insufficient.

(4) Average Insufficiency of Up-Regulation (AIUR)

AIUR is introduced to characterize the average shortage of up-regulation resources per minute, due to the mismatch of up-regulation resources and requirements [18]:

$$
E_{\text {ins }}^{\text {up }}=\frac{\sum_{t \in T_{\text {ins }}^{\text {up }}}\left|P_{\mathrm{M}}^{\mathrm{up}}(t, \tau)\right|}{T_{\text {ins }}^{\text {up }}},
$$

where the numerator represents the total insufficiency of up-regulation resource.

(5) Average Insufficiency of Down-Regulation (AIDR)

AIDR is introduced to characterize the average shortage of down-regulation resources per minute, due to the mismatch of down-regulation resources and requirements [18]: 


$$
E_{\text {ins }}^{\text {down }}=\frac{\sum_{t \in T_{\text {ins }}^{\text {down }}}\left|P_{\mathrm{M}}^{\text {down }}(t, \tau)\right|}{T_{\text {ins }}^{\text {down }}},
$$

where the numerator represents the total insufficiency of down-regulation resource.

\subsection{Adaptability of Operation State}

The adaptability of operation state refers to the ability to stabilize power disturbances, resist uncertain shocks, and maintain a safe, efficient, and stable operating state by relying on its own network structure. Aiming at the uncertain operating environment of high penetration of the renewable energy integrated system, the expected load rate and line power fluctuation rate of the line are proposed and the actual operating state of a single line is characterized from both the load level and the degree of fluctuation. Furthermore, based on the weighted entropy and joint weighted entropy theory, the operating state characteristics of each single line are integrated to obtain the overall operating state of the power system, so as to achieve a comprehensive assessment of the safety, efficiency, and stability of the actual operating state of the power system from the spatiotemporal dimension.

\subsubsection{Basic Characteristics}

\section{(1) Operational Security}

System operation security refers to the safety degree of the overall state, which can be quantitatively characterized by measuring the safety distance between the current operating state and the fault state. Self-organized critical theory is a typical evaluation method based on the above ideas [19]. The self-organized critical state represents the marginal state of the system operation. In this state, any disturbance may cause system failure or even large-scale cascading failure. Since the traditional operation mode of the power system is relatively fixed, the self-organized critical theory mainly starts from the spatial dimension and evaluates the safety of the system by analyzing the concentration and order of the load levels between lines under a certain operation mode. However, due to the superposition of the fluctuations of the renewable energy output, the load level of lines shows spatiotemporal differences. In addition, the traditional self-organized critical theory emphasizes that the lower the load rate, the safer it is and ignores the economics of operation. Therefore, in high penetration of the renewable energy integrated system, when the operating state of each line is concentrated and orderly at a reasonable load level recognized by the operator, the actual operating state of the power system is safer.

\section{(2) Operational Efficiency}

The operational efficiency refers to the economic utilization level of power system transmission equipment and reflects the reasonable degree of the power system investment, planning, and operation. If the utilization efficiency is too low, it will cause a large number of transmission equipment to be idle and redundant, indicating that the grid investment is too large and there is a lot of waste of resources. The high utilization efficiency of the power system improves the economics of power system investment and operation, but this situation often has a limited safety margin and there is a risk of safe operation. Existing operational efficiency indexes just reflect the average utilization level of transmission equipment and cannot reflect the penetration of lines with high or low utilization efficiency and the impact on overall efficiency. In addition, for the operation state with reasonable utilization efficiency, the operational efficiency of the system cannot be simply characterized by the load level. Therefore, in an uncertain operating environment where high penetration of renewable energy is integrated, operation state with good efficiency and adaptability means that each line is always operating within a reasonable range as determined by the planner. The utilization level of each line is similar, so that the overall operation state is efficient and reasonable. 


\section{(3) Operational Stability}

The stability of the operating state can be understood as the adaptability of the operation state to power fluctuations such as renewable energy or the ability to maintain the operation state in a dynamic environment. The uncertainty of the integrated renewable energy unit is coupled with the overall operation state and there will be a phenomenon where the power flow distribution is continuously shifted and the system operating state changes rapidly. At this time, if the ability to maintain the operation state is poor, it will result in a large difference in the safety and efficiency characteristics of the system state under the flow section at different times. This will not only lead to poor safety, low utilization efficiency, or heavy overloading, but also the evaluation index cannot cover the above extreme scenarios, which ultimately leads to the ambiguity of the index and reduces the accuracy of the overall assessment. In the face of various power fluctuations and shocks coupled in the operation, the operating state of the various lines has a low degree of change and the fluctuation range of the transmission power is small. The power disturbance is suppressed to the greatest extent and eventually it will not cause a huge change in the overall operating state. Therefore, when the load level of all lines is concentrated and orderly within the reasonable operating range recognized by the operating personnel, it indicates that the safety and security of the current operating state is at a good level. In other words, showing good adaptability to the uncertainty of the complex spatiotemporal distribution of renewable energy.

\subsubsection{State Evaluation Model}

\section{(1) Expected Line Load Rate}

The high frequency and large range of output power changes of renewable energy, the superimposition of spatiotemporal characteristic lead to the continuous transfer of the state. For each line, the transmission power fluctuates and changes, which can be understood as a time series. The line undertakes the corresponding power transmission task at different times according to the power flow distribution. Therefore, this paper first defines the expected line load rate to describe the average load level of the transmission power in each scenario [18]:

$$
F_{i}=\frac{\int_{-\infty}^{+\infty}\left|P_{i} \varphi\left(P_{i}\right)\right| \mathrm{d} P_{i}}{S_{i, \max }},
$$

where $P_{i}$ and $\varphi\left(P_{i}\right)$ represent the actual transmission power of line $i$ and the probability density function respectively; the numerator represents the expected value of the transmission power; $S_{i, \max }$ represents the rated transmission power capacity of the line.

\section{(2) Line Power Fluctuation Rate}

Fluctuation is a typical characteristic of high penetration of renewable energy integrated systems compared with traditional grids. The line power fluctuation rate is used to characterize the deviation of the actual transmission power of the line at different times from the average load level in each scenario. This index represents the fluctuation range of the actual transmission power from the average load level and reflects the dispersion degree of the transmission power [18]:

$$
\begin{gathered}
B_{i}=\frac{\sqrt{E\left(P_{i}^{2}\right)-E\left(P_{i}\right)^{2}}}{\min \left\{\left|S_{i, \max }-E\left(P_{i}\right)\right|,\left|E\left(P_{i}\right)\right|\right\}}, \\
E\left(P_{i}\right)=\int_{-\infty}^{+\infty} P_{i} \varphi\left(P_{i}\right) \mathrm{d} P_{i} \\
E\left(P_{i}^{2}\right)=\int_{-\infty}^{+\infty} P_{i}^{2} \varphi\left(P_{i}\right) \mathrm{d} P_{i}
\end{gathered}
$$


where $E(\cdot)$ is the expected value of the relevant parameters; the numerator is the fluctuation value of the transmission power; the denominator is the allowable power fluctuation range, i.e., the power fluctuation capacity.

\subsubsection{Evaluation Indexes}

The above evaluation model can characterize the safety margin, efficiency level, and stability of each line, the actual operating state should be an overall evaluation of all lines, and the operating state of each line will constitute the overall operating state. Therefore, based on the weighted entropy [20] and joint weighted entropy theory [21], this paper proposes adaptability evaluation indexes for the operation state of overall system.

\section{(1) Weighted Entropy of Expected Line Load Rate (WEELLR)}

The operating state is regarded as the system as a whole and each load rate interval is regarded as a system event. The ratio of the number of lines in each interval to the total number of lines is regarded as the probability of occurrence of events in this interval. The weights of each interval are subjectively determined according to planners, and set the optimal operating interval weight to the minimum. The weighted entropy theory can be used to establish WEELLR to evaluate the concentration and rationality of the operating state distribution.

The load rate interval is expressed as a set $M=\left[M_{1}, M_{2}, \ldots, M_{5}\right]$, where interval $M_{1}$ indicates that the load rate interval is $\left(0,20 \%\right.$ ] of the rated capacity and so on, $\mathrm{M}_{5}$ indicates $(80 \%, 100 \%]$. $N_{\mathrm{M} i}$ is the number of lines whose expected load rate belongs to the interval. Then, the probability that the line is in the $i$ th expected load rate interval can be expressed as [20]:

$$
p\left(F_{\mathrm{M}_{i}}\right)=\frac{N_{\mathrm{M}_{i}}}{\sum_{i=1}^{5} N_{\mathrm{M}_{i}}} .
$$

The weight of the expected load rate interval of each line $\omega\left(F_{\mathrm{M} i}\right)$ can be determined according to the planner's comprehensive consideration of the grid efficiency and safety margin. For example, the rational ranking of the load rate interval is: $\mathrm{M}_{3}>\mathrm{M}_{2}>\mathrm{M}_{1}>\mathrm{M}_{4}>\mathrm{M}_{5}$, where the " $>$ " symbol indicates that the left side of the symbol is more reasonable than the right-side object. The above sorting method shows that the planner thinks that when the line load rate in $(80 \%, 100 \%]$ of the rated capacity is the most reasonable and its weight is the smallest. Therefore, WEELLR is expressed as [20]:

$$
H_{\mathrm{F}}=-\sum_{i=1}^{5} \omega\left(F_{\mathrm{M}_{i}}\right) p\left(F_{\mathrm{M}_{i}}\right) \ln p\left(F_{\mathrm{M}_{i}}\right) .
$$

This index reflects the concentration and rationality of the distribution of the average load levels between lines from the spatial dimension. The smaller the index value, the more the expected load rate of the line is concentrated in the range that the operator believes is more reasonable. The overall average state has both safety and efficiency and has good adaptability.

\section{(2) Weighted Entropy of Line Power Fluctuation Rate (WELPFR)}

Due to fluctuations of renewable energy output, the operating state will shift from the average state. When the offset is large, it will cause the safety and efficiency of the running state to not maintain the average state. Therefore, WELPFR is introduced to quantify the degree of this deviation and to evaluate the stability of the system operating state.

The operating state is still regarded as the system as a whole and each power fluctuation rate interval is regarded as a system event. The ratio of the number of lines existing in each interval to the total number of lines is regarded as the probability of occurrence of events in this interval. Because 
the smaller the line power fluctuation, the more stable the overall system state, so the weight of the operating interval with the smallest power fluctuation rate is set to the minimum. The weighted entropy theory can be used to establish WELPFR to evaluate the stability of the grid operating state distribution. The power fluctuation rate interval is expressed as a set $V=\left[V_{1}, V_{2}, \ldots, V_{10}\right]$, where interval $\mathrm{V}_{1}$ represents the power fluctuation rate value interval is $(0,10 \%]$ and so on, $\mathrm{V}_{10}$ indicates $(90 \%, 100 \%] . N_{\mathrm{V} j}$ is the number of lines whose line power fluctuation rate belongs to the interval $\mathrm{V}_{j}$. Then, the probability that the line is in the $j$ th power fluctuation rate interval can be expressed as [20]:

$$
p\left(B_{\mathrm{V}_{j}}\right)=\frac{N_{\mathrm{V}_{j}}}{\sum_{j=1}^{10} N_{\mathrm{V}_{j}}}
$$

The smaller the power fluctuation of each line is, the more stable the system is. Therefore, the smaller the weight of the power fluctuation rate interval, the higher the rationality and the average value of the line power fluctuation rate of each interval can be directly used as the weight of the interval. Let $k$ denote the line whose line power fluctuation rate is in the interval $\mathrm{V}_{j}$, the weights is calculated as follows:

$$
\omega\left(B_{\mathrm{V}_{j}}\right)=\frac{1}{N_{\mathrm{V}_{j}}} \sum_{k \in \mathrm{V}_{j}} B_{k}
$$

Thus, WELPFR can be expressed as:

$$
H_{\mathrm{B}}=-\sum_{j=1}^{10} \omega\left(B_{\mathrm{V}_{j}}\right) p\left(B_{\mathrm{V}_{j}}\right) \ln p\left(B_{\mathrm{V}_{j}}\right) .
$$

This index considers the fluctuation of the transmission power of all lines and can reflect the stability operating state from the time and space dimensions. The smaller the index value, the more the line power fluctuation range is smaller, power system has better adaptability and smoothing ability to the renewable energy power fluctuation, and shows high inertia operating characteristics.

\section{(3) Joint Weighted Entropy of Operation State (JWEOS)}

WEELLR evaluates the safety and efficiency operating state, WELPFR evaluates the stability of the operating state. The above two indexes and three attributes jointly determine the adaptability and rationality of the system operating state. However, in practical applications, it is often more desirable to use an index to directly assess the adaptability and rationality of the operation state. Therefore, this paper combines the two indexes and further propose JWEOS to comprehensively evaluate the safety, efficiency, and stability of operation state.

The operating state of high penetration of the renewable energy integrated system can be characterized by the expected line load rate and line power fluctuation rate. If the grid operating state is regarded as the whole system, the expected line load rate and line power fluctuation rate can be regarded as the two types of events that constitute the overall system, the concentration and rationality jointly determine the adaptability operating state. Because, under the considered operation scenario and corresponding operation mode, the expected load rate and power fluctuation rate distribution are obtained through simulation. Therefore, JWEOS is formulated as [21]:

$$
H_{\mathrm{FB}}=-\sum_{i=1}^{5} \sum_{j=1}^{10} \omega\left(F_{\mathrm{M}_{i}}\right) \omega\left(B_{\mathrm{V}_{j}}\right) p\left(F_{\mathrm{M}_{i}}\right) p\left(B_{\mathrm{V}_{j}}\right) \ln \left[p\left(F_{\mathrm{M}_{i}}\right) p\left(B_{\mathrm{V}_{j}}\right)\right] .
$$

The smaller the index value, the more lines in the system are operating within the load level interval that the planner thinks reasonable and the more line with smaller power fluctuation range. Combined with the definition of adaptability of operation state, it can be seen that the system considers 
both safety and efficiency in this operating state, with strong robustness and a comprehensive high level of adaptability.

\subsection{Adaptability of Network Structure}

The adaptability of network structure means that in an uncertain operating environment where high penetration of renewable energy is connected to the grid, the power system will ultimately improve the ability components and the overall structure to withstand uncertain impacts by optimizing the network topology and reducing weak links. According to the complex network theory and vulnerability theory, bus and branch electric betweenness indexes are used to characterize the importance and robustness of a single component [22]. Based on the uniformity theory, weighted entropy and joint weighted entropy theory, the impact of buses and branches on the topology is integrated. From a single element to the overall power system, the weak links are identified and the ability of the overall network structure to withstand uncertain shocks.

\subsubsection{Basic Characteristics}

In the power system analysis model, buses and branches are the most basic components that make up the network structure. The rationality of the connection relationship between buses and branches will directly determine the ability of the network structure to resist the impact of uncertain factors. In the context of high penetration of the renewable energy integrated system, if there are weak buses or branches, it is very easy to cause large-scale cascading failures from renewable energy power shocks. Therefore, evaluating the robustness of buses and branches is the key to understanding the ability of the overall structure to withstand uncertainties.

\section{(1) Bus Robustness}

On the one hand, bus robustness is determined by the load level and the integrated generator capacity, on the other hand, by the spatial position of bus in the power system topology. Bus that bears important loads or bus with important generators often has a high importance level and needs to pay special attention to ensure reliable power supply of the critical load. Bus with more complicated contact relationships in the system also tends to have a higher importance level. Once this type of bus withdraws due to various uncertain shocks or reduces its contribution to the operation, it has a wide range of coverage and is often easy to cause larger failures. Therefore, bus with higher criticality tends to become weak links, it is of great significance to improve the robustness of the network structure to identify the key bus and reduce its weakness through reasonable planning.

\section{(2) Branch Robustness}

The transmission line assumes the key role of connecting various types of generator and load buses, which is the basic platform to ensure the reliable power supply. On the one hand, branch robustness is determined by the importance of buses connected by the transmission corridor, on the other hand, by the transmission capacity undertaken by the transmission corridor in the system power supply and demand balance. The transmission lines with more complicated contact relationships and contact buses belonging to important buses often have higher importance levels. Once such lines exit the system due to various uncertain impacts, it is easy to cause large-scale chain failures and large-scale power outages. Therefore, identifying the weak links of the power transmission line and on this basis, reducing the number of weak links and reducing the degree of weakness is the key to improving the robustness of network structure.

\subsubsection{State Evaluation Model}

Complex network theory is an abstract description of complex systems composed of different nonlinear basic units. Its core idea is to abstract complex systems into complex networks composed of 
buses and branches and use graph theory, statistical theory, etc. The classic index of the method to quantify the network structure characteristics of complex systems. Among them, buses of the complex network represent the basic units that make up the system and branches reflect the association between the basic units. In the analysis of complex networks in power systems, the electric betweenness is the most commonly used. This index is based on the network intermediary index and combined with the improvement of the actual characteristics of the power flow distribution. It is divided into electric betweenness of buses and branches. Then, clarify the network's weak links and their weaknesses. It means that any power generation and load bus pair in the power system is selected, the power generation and load power of the bus pair are used as power weights and then the unit current is added between bus pairs and Kirchhoff's law solves the current in the entire network After the traversal considers all bus pairs, the current through a bus or branch is weighted and accumulated and the electric betweenness of bus or branch can be obtained. The index can reflect the power capacity of the different power generation and load bus pairs by adjusting the weights and can accurately characterize the occupancy of each bus and branch element based on Kirchhoff's theorem.

\section{(1) Electric Betweenness of Bus}

Electric betweenness of bus characterizes the occupancy of all bus components by each power transmission path of the grid, i.e., the contribution of different bus in the power transmission. The larger the electric betweenness of bus, the greater the contribution and importance of bus in the power transmission of the whole network and the more easily and disturbed the system power is. By obtaining the electric betweenness values of all buses in the power system, the weak ranking of buses can be obtained. The electric betweenness of bus $i$ can be calculated as [22]:

$$
D_{i}=\sum_{a \in \mathbf{G e}, b \in \mathbf{L o}} \sqrt{\omega_{a} \omega_{b}} B_{e, a b}(i),
$$

where $a, b$ respectively represent the number of generator and load bus; Ge and Lo represent the set of generator and load bus; $\omega_{a}, \omega_{b}$ represent the weights of generator and load bus, their values are denoted as the actual power; $B_{e, a b}(i)$ is the current distribution of bus $i$, after add a unit current element between pairs of generator load bus $(a, b)$, which can be calculated as [22]:

$$
B_{e, a b}(i)=\left\{\begin{array}{lr}
\frac{1}{2} \sum_{j}\left|I_{a b}(i, j)\right| & i \neq a, b \\
1 & i=a, b
\end{array},\right.
$$

where $I_{a b}(i, j)$ is the current of branches $(i, j)$ after adding the unit current element between the generator load bus pair $(a, b)$ and bus $j$ represents the remaining buses directly connected to bus $i$. When bus $i$ is one of the generator load bus pairs, the current value passing through bus is regarded as the unit current.

\section{(2) Electric Betweenness of Branch}

Electric betweenness of branch characterizes the occupancy of all power system components by each power transmission line, i.e., the contribution of each branch in the power transmission of the entire network. The greater the electric betweenness of branch, the greater the contribution and importance of branch in the power transmission. It also indicates that branch is more susceptible to system power disturbances and has less robustness. The easier it is to become the weak link in the overall grid. The electric betweenness of branch $m$ can be calculated as [22]:

$$
L_{m}=\sum_{a \in \mathbf{G e}, b \in \mathbf{L o}} \sqrt{\omega_{a} \omega_{b}}\left|I_{a b}(m)\right|,
$$

where $I_{a b}(m)$ is the current of branch $m$ after the unit current element is added between the generator load bus pair $(a, b)$. 


\subsubsection{Evaluation Indexes}

(1) Weighted Entropy for Electric Betweenness of Bus (WEEBBus)

Although the electric betweenness of bus can clarify the order of the weakness of each bus within the power system, it cannot compare the robustness of different network structures from the perspective of the overall bus. Therefore, this paper proposes WEEBBus to clarify the difference in robustness of different network structures. The network topology is regarded as the system, the electric betweenness interval of each bus is regarded as event. The ratio of the number of buses in each interval to the total number of buses is regarded as the probability of occurrence of events in this interval. Since the smaller the electric betweenness of bus, the more robust the component and the overall structure, the interval weight with the smallest electric betweenness is set to the minimum. Thus, the weighted entropy theory can be used to establish WEEBBus and the robustness of network structure can be evaluated from the perspective of bus.

Represent the interval for electric betweenness of bus as a set $\mathrm{O}=\left[\mathrm{O}_{1}, \mathrm{O}_{2}, \ldots, \mathrm{O}_{5}\right], N_{\mathrm{O} i}$ is the number of bus whose electric betweenness is in the interval $\mathrm{O}_{i}$. Then, the probability that the electric betweenness of bus belongs to the $i$ th interval $\mathrm{O}_{i}$ can be expressed as [20]:

$$
p\left(D_{\mathrm{O}_{i}}\right)=\frac{N_{\mathrm{O}_{i}}}{\sum_{i=1}^{5} N_{\mathrm{O}_{i}}} .
$$

The smaller the electric betweenness of bus, the less the weak links of the network, the better the robustness of the structure. Therefore, the smaller the electric betweenness value is, the smaller the weight value of interval is. Here, let $k$ denote bus whose electric betweenness belongs the interval $\mathrm{O}_{i}$, the average value of the electric betweenness for each interval is used as the weight value of the interval [20]:

$$
\omega\left(D_{\mathrm{O}_{i}}\right)=\frac{1}{N_{\mathrm{O}_{i}}} \sum_{k \in \mathrm{O}_{i}} D_{k}
$$

Thus, WEEBBus can be formulated as [20]:

$$
H_{\mathrm{D}}=-\sum_{i=1}^{5} \omega\left(D_{\mathrm{O}_{i}}\right) p\left(D_{\mathrm{O}_{i}}\right) \ln p\left(D_{\mathrm{O}_{i}}\right) .
$$

The smaller the index value, the more buses in the power system are concentrated in a smaller value interval. This shows that from the perspective of buses, the overall structure is balanced, robust, and has few weak links and is not easily affected by power disturbances. Network structure has higher adaptability and stronger ability to withstand uncertain shocks.

(2) Weighted Entropy for Electric Betweenness of Branch (WEEBBra)

When the electric betweenness of all branches is concentrated in a relatively small range, it indicates that there is no weak link in the branch of the network structure. The topological structure is regarded as the system as a whole, the electric betweenness interval of each branch is regarded as a system event and the ratio of the number of branches in each interval to the total number of branches is regarded as the probability of occurrence of events in this interval. The interval weight with the smallest electric betweenness is set to the minimum and so on. In this way, the weighted entropy theory can be used to establish WEEBBra and the robustness of the network structure can be evaluated from the perspective of the branch. 
Represent the interval for electric betweenness of branch as a set $Z=\left[Z_{1}, Z_{2}, \ldots, Z_{5}\right], N_{Z j}$ is the number of branches whose electric betweenness belongs to interval $Z_{j}$. Then, the probability that the electric betweenness of bus belongs to the $j$ th interval $Z_{j}$ can be expressed as [20]:

$$
p\left(L_{Z_{j}}\right)=\frac{N_{Z_{j}}}{\sum_{j=1}^{5} N_{Z_{j}}} .
$$

On this basis, determine the weight $\omega\left(L_{Z i}\right)$ of the interval for electric betweenness of each branch. Similarly, the smaller the electric betweenness is, the smaller the weight setting. Let $k$ denote the branch whose electric betweenness belong the interval $Z_{j}$, the average value of electric betweenness of the branch for each interval is used as the weight of the interval [20]:

$$
\omega\left(L_{Z_{j}}\right)=\frac{1}{N_{Z_{j}}} \sum_{k \in Z_{j}} L_{k}
$$

Thus, WEEBBra can be formulated as [20]:

$$
H_{\mathrm{L}}=-\sum_{j=1}^{5} \omega\left(L_{\mathrm{Z}_{j}}\right) p\left(L_{\mathrm{Z}_{j}}\right) \ln p\left(L_{\mathrm{Z}_{j}}\right)
$$

The smaller the index, the more branches are concentrated in the smaller value interval of electric betweenness. This shows that from the perspective of branch circuit, the overall structure is balanced, robust, and not easily affected by power disturbances. Network structure has higher adaptability and stronger ability to withstand uncertain shocks.

(3) Joint Weighted Entropy of Network Structure (JWENS)

From the above analysis, we can see that buses and branches are the most basic components of the power network and their respective robustness will directly affect the ability of the overall structure to withstand uncertain shocks. Although the above two weighted entropy indexes can describe the robustness of all buses or all branches separately, they cannot describe the robustness of the overall structure. Therefore, this paper further proposes JWENS. Based on the overall consideration of all the basic components, the robustness topology is comprehensively evaluated and then the adaptability of network structure to uncertain shocks is reflected.

The most basic components topology includes two types of buses and branches. If the power system topology is regarded as the system as a whole, then buses and branches can be regarded as two types of events that constitute the system as a whole. The rationality together determines the adaptability of the network structure. For a certain network structure, the connection relationship between its internal buses and branches is fixed and the distribution of electric betweenness of buses and branches is also independent and determined. Therefore, the joint weighted entropy model of the decoupling calculation is used to establish JWENS as follows [21]:

$$
H_{\mathrm{DL}}=-\sum_{i=1}^{5} \sum_{j=1}^{5} \omega\left(D_{\mathrm{O}_{i}}\right) \omega\left(L_{\mathrm{Z}_{j}}\right) p\left(D_{\mathrm{O}_{i}}\right) p\left(L_{\mathrm{Z}_{j}}\right) \ln \left[p\left(D_{\mathrm{O}_{i}}\right) p\left(L_{\mathrm{Z}_{j}}\right)\right]
$$

The smaller the index value, the more buses and branches have smaller electric betweenness. In other words, the fewer weak links in system, the smaller the impact of power disturbance, the better the overall robustness, and the higher the adaptability to uncertain shocks. 


\section{Adaptable Source-Grid Expansion Planning Model}

From the source side, the upper limit of the system regulation capability is determined by the capacity and regulation rate of the power supply regulation resources. However, due to the limitation of network transmission, the actual regulation capacity of the system is often less than the regulation capacity of the source. In the process of power supply and demand balance, although it does not provide or consume regulation resources in essence, the power system plays a supporting role in the process of supply and demand balance. Therefore, the establishment of a grid structure that is compatible with the process of supply and demand distribution of source and load power can ensure the full utilization and efficient transmission of regulation resources and maximizes the release of system regulation capabilities.

From the grid side, the transmission network not only needs to support the basic task of power supply and demand balance, but also needs to maintain excellent operation state and resist renewable energy during the above process. The impact of energy power fluctuations on the operating state ensures the safety, stability, and efficiency of the operating state. On the one hand, the state depends on the reasonable degree of network structure, on the other hand, it is also greatly affected by the power supply and system operation mode. Therefore, the establishment of a network structure that is compatible with power supply transformation and operation schemes is also the key to ensuring an efficient and reliable actual operating state and improving the adaptability of network structure.

Based on the above analysis, it is known that designing mutually source-grid coordinated planning schemes is the key to comprehensively improving the adaptability of high penetration of renewable energy integrated systems. Therefore, this paper proposes a two-stage expansion planning method. In the first stage, the current supply and demand balance of the system is evaluated. If the supply and demand balance adaptability meet the threshold preset by the planner, then the power supply does not need to be upgraded and directly entered into the second stage and the power system is adapted through the adaptable grid planning model planning and reconstruction to optimize the structure and operation state. If the supply and demand balance adaptability index does not meet the threshold set by the planner, the first stage adopts the adaptable source planning model to upgrade the technical parameters of the existing units. Then, the optima source plan is utilized as input in the second stage planning. On this basis, the adaptable grid planning model is applied to obtain the optimal grid plan. The details are as follows.

\subsection{Obective Funuction}

\subsubsection{Adaptable Source Expansion Planning Model}

The objection function is to minimize the comprehensive cost of source transformation, which includes transformation cost for the quick regulation unit, curtailment cost for renewable energy power, and load shedding cost, as shown below:

$$
\begin{gathered}
\min F=C_{\mathrm{G}}+C_{\mathrm{WR}}+C_{\mathrm{WL}}, \\
C_{\mathrm{G}}=\left(k_{1}+k_{2}\right) \sum_{i \in \Omega_{\mathrm{G}}} z_{i} \cdot\left(c_{1, i} x_{i}+c_{2, i} y_{i}\right), \\
k=\frac{r(1+r)^{n}}{(1+r)^{n}-1}, \\
C_{\mathrm{WR}}=D \sum_{s \in \mathbf{S}} p(s) \sum_{t \in \mathrm{T}_{\text {ins }}^{\text {down }}} c_{\mathrm{REG}} \cdot\left|P_{\mathrm{M}}^{\text {down }}(t, \tau) \cdot \tau\right|, \\
C_{\mathrm{WL}}=D \sum_{s \in \mathbf{S}} p(s) \sum_{t \in \mathrm{T}_{\text {ins }}^{\mathrm{up}}} c_{\mathrm{LOAD}} \cdot P_{\mathrm{M}}^{\mathrm{up}}(t, \tau) \cdot \tau \mid
\end{gathered}
$$


where $C_{\mathrm{G}}$ is the cost of regulation capacity transformation, $C_{\mathrm{WR}}$ is the annual cost of renewable energy generation, $C_{\mathrm{WL}}$ is the annual load shedding cost; $k_{1}, k_{2}$ are the fund recovery factor and the project fixed operating rate, which are used to allocate the one-time investment cost to each year of life span, $r$ is the discount rate, $n$ is the economically applicable year of project; $\Omega_{\mathrm{G}}$ is set of fast regulating units; $z_{i}$ is the sign for transformation, $x_{i}$ is the transformation capacity of unit, $y_{i}$ is the transformation quantity of regulation rate; $c_{1, i}$ is the unit cost for capacity transformation, $c_{2, i}$ is unit cost for transformation of regulation rate; $D$ is the number of days of operation per year, $S$ is the set for typical scenarios of renewable energy outputs, $p(s)$ is the probability of typical scenarios $s, c_{\text {REG }}$ is the unit cost of renewable energy curtailment, and $T_{\text {ins }}{ }^{\text {down }}$ is the time of renewable energy curtailment; $\left|P_{\mathrm{M}}{ }^{\text {down }}(t, \tau) \cdot \tau\right|$ is the curtailment power of the renewable energy, $c_{\mathrm{LOAD}}$ is the cost of load shedding, $T_{\mathrm{ins}}$ up is the time of load shedding, $\left|P_{\mathrm{M}}{ }^{\text {up }}(t, \tau) \cdot \tau\right|$ is the power of load shedding.

\subsubsection{Adaptable Grid Expansion Planning Model}

Considering the economy of grid planning, adaptability of operation state, and adaptability of network structure, the objective function is to minimize the cost of grid expansion, joint weighted entropy index of operation state, and joint weighted entropy index of network structure. Due to the dimensional differences between the various objectives, the objection function is expressed as a multi-objective function in vector form:

$$
\begin{gathered}
\min F=\min \left(C_{\mathrm{N}}, H_{\mathrm{FB}}, H_{\mathrm{DL}}\right), \\
C_{\mathrm{N}}=\left(k_{1}+k_{2}\right) \sum_{i \in \Omega_{1}} c_{i} n_{i} l_{i} \\
H_{\mathrm{FB}}=-\sum_{i=1}^{5} \sum_{j=1}^{10} \omega\left(F_{\mathrm{M}_{i}}\right) \omega\left(B_{\mathrm{V}_{j}}\right) p\left(F_{\mathrm{M}_{i}}\right) p\left(B_{\mathrm{V}_{j}}\right) \ln \left[p\left(F_{\mathrm{M}_{i}}\right) p\left(B_{\mathrm{V}_{j}}\right)\right] \\
H_{\mathrm{DL}}=-\sum_{i=1}^{5} \sum_{j=1}^{5} \omega\left(D_{\mathrm{O}_{i}}\right) \omega\left(L_{\mathrm{Z}_{j}}\right) p\left(D_{\mathrm{O}_{i}}\right) p\left(L_{\mathrm{Z}_{j}}\right) \ln \left[p\left(D_{\mathrm{O}_{i}}\right) p\left(L_{\mathrm{Z}_{j}}\right)\right]
\end{gathered}
$$

where $C_{\mathrm{N}}$ is the cost of grid expansion; $H_{\mathrm{FB}}$ is JWEOS; $H_{\mathrm{DL}}$ is JWENS; $\Omega_{1}$ is the set of expanded lines, $c_{i}$ is the unit investment of reconstructed line, $n_{i}$ is the circuit number of expanded lines, $l_{i}$ is the total length of the reconstructed line.

\subsection{Constraints}

\subsubsection{Constraints for Source Expansion Planning}

The constraints of adaptable source expansion planning model include power balance constraints (39), unit output constraints (40) and (41), unit ramp constraints (42), power flow constraints (43), and insufficient rate index of supply and demand balance constraints (44). The specific formulas are as follows.

$$
\begin{aligned}
& \sum_{i \in \Omega_{\mathrm{g}}} P_{i, \mathrm{~g}}(s, t)+\sum_{i \in \Omega_{\mathrm{G}}} P_{i, \mathrm{G}}(s, t)+\sum_{i \in \Omega_{\mathrm{REG}}} P_{i, \mathrm{REG}}(s, t)=\sum P_{\mathrm{L}}(s, t), \\
& P_{i, \mathrm{G}}^{\min } \leq P_{i, \mathrm{G}}(s, t) \leq P_{i, \mathrm{G}}^{\max }, i \in \Omega_{\mathrm{G}}, \\
& P_{i, \mathrm{~g}}^{\min } \leq P_{i, \mathrm{~g}}(s, t) \leq P_{i, \mathrm{~g}}^{\max }, i \in \Omega_{\mathrm{g}}, \\
& -R_{i, \mathrm{G}}^{\mathrm{down}} \cdot \tau \leq P_{i, \mathrm{G}}(s, t)-P_{i, \mathrm{G}}(s, t-\tau) \leq R_{i, \mathrm{G}}^{\mathrm{up}} \cdot \tau, i \in \Omega_{\mathrm{G}}, \\
& \boldsymbol{-} \boldsymbol{B} \boldsymbol{\theta}(s, t)+\boldsymbol{P}_{\mathrm{g}}(s, t)+\boldsymbol{P}_{\mathrm{G}}(s, t)+\boldsymbol{P}_{\mathrm{REG}}(s, t)=\boldsymbol{P}_{\mathrm{L}}(s, t), \\
& R_{\text {ins }} \leq \alpha,
\end{aligned}
$$


where $\Sigma P_{\mathrm{L}}(s, t)$ is the total load at time slot $t$ in scenario $s ; P_{i, \mathrm{~g}}(s, t), P_{i, \mathrm{G}}(s, t)$, and $P_{i, \mathrm{REG}}(s, t)$ are the actual output power of the conventional unit, the fast regulating unit, and the renewable energy unit at time slot $t$ in scenario $s ; \Omega_{\mathrm{g}}, \Omega_{\mathrm{G}}, \Omega_{\mathrm{REG}}$ are the sets of the corresponding units; $P_{i, \mathrm{G}}{ }^{\max }$ and $P_{i, \mathrm{G}}{ }^{\min }$ are the upper and lower limits for the output power of fast regulating unit; $P_{i, \mathrm{~g}}{ }^{\max }$ and $P_{i, \mathrm{~g}}{ }^{\mathrm{min}}$ are the upper and lower limits for the output power of the conventional unit; $R_{i, \mathrm{G}}{ }^{\text {up }}$ and $R_{i, \mathrm{G}}{ }^{\text {down }}$ are the maximum ramp up and down rate of the fast-regulation unit under the time scale $\tau ; B$ is the admittance matrix; $\theta$ is the voltage phase angle vector; $P_{i}$ is the transmission power of line $i ; S_{i, \max }$ is the rated transmission capacity of line $i ; R_{\text {ins }}$ is the insufficient rate index of supply and demand balance; $\alpha$ is the set threshold value.

\subsubsection{Constraints for Grid Expansion Planning}

The constraints of adaptable grid expansion planning model include the integer constraint for the circuit number of new lines, the rated conditions, the $\mathrm{N}-1$ conditions, and the safe operation constraints in each scenario. The specific formulas are as follows.

$$
\begin{gathered}
n_{i}^{\min } \leq n_{i} \leq n_{i}^{\max }, i \in \Omega_{\mathrm{l}} n_{i} \in \mathbf{Z}, \\
\left\{\begin{array}{l}
-\mathbf{B} \boldsymbol{\theta}+\boldsymbol{P}_{\mathrm{g}}+\boldsymbol{P}_{\mathrm{G}}+\boldsymbol{P}_{\mathrm{REG}}=\boldsymbol{P}_{\mathrm{L}} \\
\left|P_{i}\right| \leq S_{i, \max }, i \in \Omega_{\mathrm{L}} \\
P_{i, \mathrm{~g}}^{\min } \leq P_{i, \mathrm{~g}} \leq P_{i, \mathrm{~g}}^{\max }, i \in \Omega_{\mathrm{g}} \\
P_{i, \mathrm{G}}^{\min } \leq P_{i, \mathrm{G}} \leq P_{i, \mathrm{G}}^{\max }, i \in \Omega_{\mathrm{G}}
\end{array}\right. \\
\left\{\begin{array}{l}
-\boldsymbol{B}^{\mathrm{N}-1} \theta^{\mathrm{N}-1}+\boldsymbol{P}_{\mathrm{g}}^{\mathrm{N}-1}+\boldsymbol{P}_{\mathrm{G}}^{\mathrm{N}-1}+\boldsymbol{P}_{\mathrm{REG}}^{\mathrm{N}-1}=\boldsymbol{P}_{\mathrm{L}}^{\mathrm{N}-1} \\
\left|P_{i}^{\mathrm{N}-1}\right| \leq S_{i, \max }, i \in \Omega_{\mathrm{L}} \\
P_{i, \mathrm{~g}}^{\min } \leq P_{i, \mathrm{~g}}^{\mathrm{N}-1} \leq P_{i, \mathrm{~g}}^{\max }, i \in \Omega_{\mathrm{g}} \\
P_{i, \mathrm{G}}^{\min } \leq P_{i, \mathrm{G}}^{\mathrm{N}-1} \leq P_{i, \mathrm{G}}^{\max }, i \in \Omega_{\mathrm{G}}
\end{array}\right. \\
\left\{\begin{array}{c}
-\boldsymbol{B} \boldsymbol{\theta}(s, t)+\boldsymbol{P}_{\mathrm{g}}(s, t)+\boldsymbol{P}_{\mathrm{G}}(s, t)+\boldsymbol{P}_{\mathrm{REG}}(s, t)=\boldsymbol{P}_{\mathrm{L}}(s, t) \\
\left|P_{i}(s, t)\right| \leq S_{i, \max }, i \in \Omega_{\mathrm{L}} \\
P_{i, \mathrm{~g}}^{\min } \leq P_{i, \mathrm{~g}}(s, t) \leq P_{i, \mathrm{~g}}^{\max }, i \in \Omega_{\mathrm{g}} \\
P_{i, \mathrm{G}}^{\min } \leq P_{i, \mathrm{G}}(s, t) \leq P_{i, \mathrm{G}}^{\max }, i \in \Omega_{\mathrm{G}} \\
-R_{i, \mathrm{G}}^{\mathrm{down}} \cdot \tau \leq P_{i, \mathrm{G}}(s, t)-P_{i, \mathrm{G}}(s, t-\tau) \leq R_{i, \mathrm{G}}^{\mathrm{up}} \cdot \tau, i \in \Omega_{\mathrm{G}}
\end{array}\right.
\end{gathered}
$$

where $n_{i}{ }^{\min }, n_{i}{ }^{\max }$ are the upper and lower limits of the circuit number of lines; the superscript " $\mathrm{N}-1$ " represents the variable in the case of $\mathrm{N}-1$ condition.

\subsection{Model Solution}

The proposed adaptable source-grid expansion planning model consists of two-stage planning. The source expansion planning model is a single objective mixed integer linear programming problem [23], which can be easily solved by a commercial solver, the Yalmip toolkit with CPLEX solver is utilized in this paper. The gird expansion planning model is a multi-objective mixed integer nonlinear programming problem, the multi-objective particle swarm optimization algorithm is utilized to search the pareto non-dominated solution set, the data envelopment analysis and analytic hierarchy process (DEAHP) decision method is used to comprehensively evaluate the optimal solution set to obtain the final optimal plan [24]. The flowchart of the detail process is shown in Figure 3. 


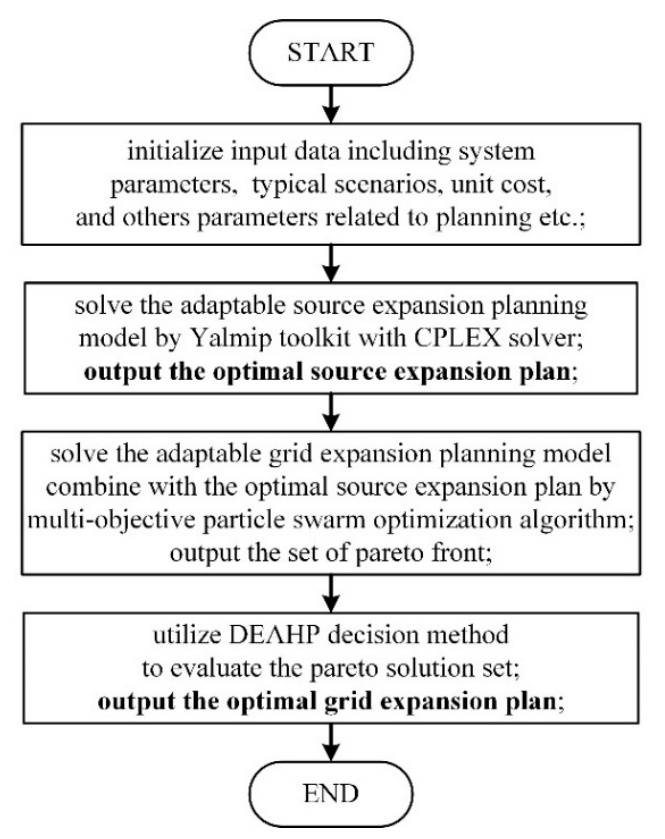

Figure 3. Flow chart of grid-generator coordination planning.

\section{Results and Discussion}

\subsection{Basic Parameters}

The Garver-18 bus power transmission system is used as a test system [25]. The network structure is shown in Figure 4. The remaining parameters are set as follows: a centralized wind farm is integrated at bus 16 and a centralized photovoltaic power plant is integrated at bus 14 . The installed capacity of renewable energy unit is $20 \%$ of the conventional unit. The typical output scenario is given in Figures 5 and 6; the system runs for 360 days, totaling 8640 h; the threshold of insufficient rate index of supply and demand balance is set as $10 \%$; the investment cost of transmission line construction is set to 800,000 yuan $/ \mathrm{km}$; the discount rate is set to $10 \%$; the service life of the project is set to 15 years; the fixed operation rate of the project is set as $5 \%$.

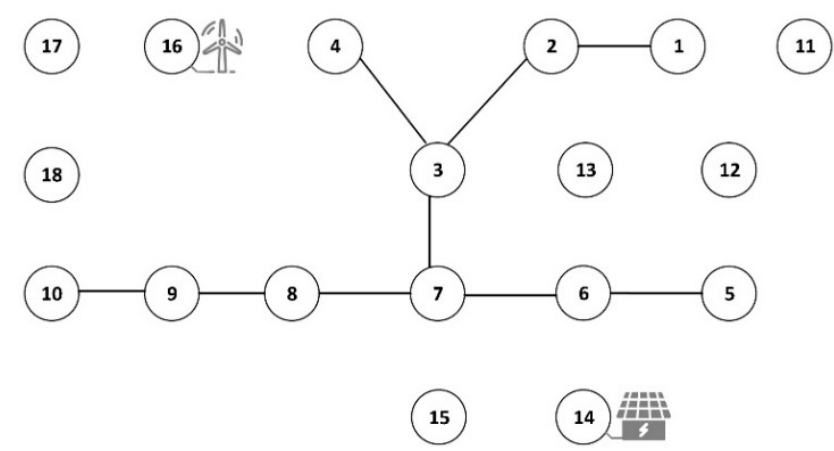

Figure 4. Garver-18 testing system. 


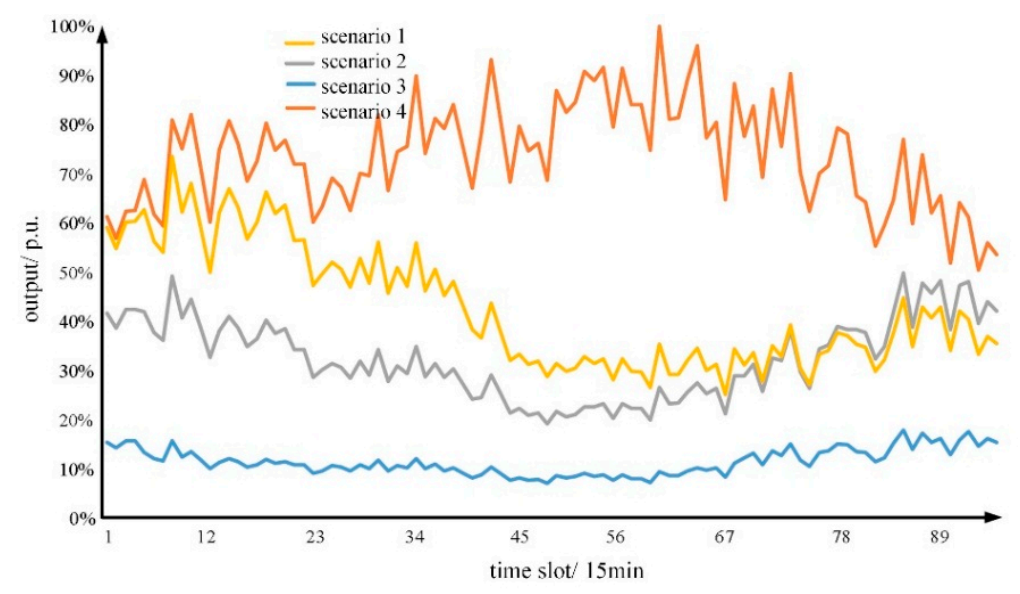

Figure 5. Typical scenarios of a wind farm.

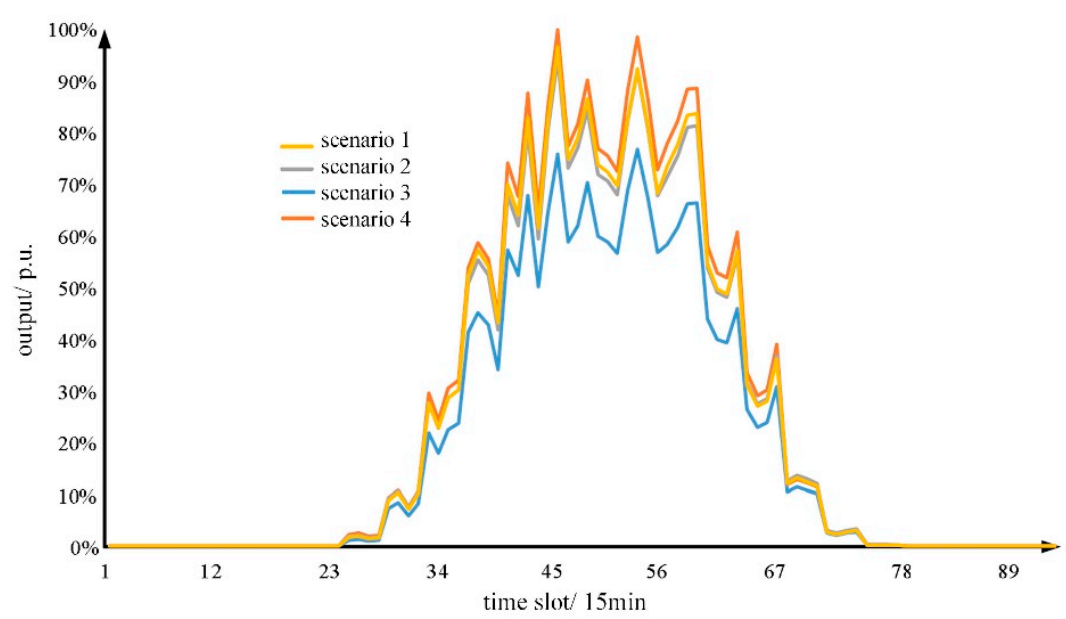

Figure 6. Typical scenarios of a photovoltaic power plant.

\subsection{Model Validation}

By solving the proposed adaptable source-grid two-stage expansion planning model, the optimal source-grid planning scheme is given in Table 1, and the economic optimal plan is also given in the last column as comparison.

Table 1. Optimal adaptable source-grid planning scheme.

\begin{tabular}{ccccc}
\hline \multirow{2}{*}{$\begin{array}{c}\text { Integrated Bus of } \\
\text { Conventional Unit }\end{array}$} & \multirow{2}{*}{$\begin{array}{c}\text { Expanded Capacity of } \\
\text { Ramp Rate/MW.15 } \text { min }^{-1}\end{array}$} & $\begin{array}{c}\text { Expanded Circuit } \\
\text { Number of Lines }\end{array}$ & $\begin{array}{c}\text { Comprehensive } \\
\text { Optimal Plan }\end{array}$ & Economic Optimal Plan \\
\hline 2 & 7.6 & & $1-2,3-7,4-7,4-16,6-13$, & $1-11,4-16,5-12,6-13,6-14,7-8$, \\
5 & 6.4 & 1 & $7-13,7-15,9-16,10-18$, & $7-15,9-16,10-18,11-12,16-17 ;$ \\
10 & 6.1 & & $11-12,11-13,16-17 ;$ & \\
11 & 5.5 & 2 & $1-11,5-12,6-14,7-8,14-15 ;$ & $6-7,7-13,8-9,9-10,14-15,17-18$ \\
14 & 5.8 & 3 & $8-9,17-18$, & $\backslash$ \\
16 & 5.9 & 4 & $9-10$ & 1 \\
\hline
\end{tabular}

\subsubsection{Optimal Adaptable Source-Grid Plan}

From the optimal source planning results in Table 1, it can be seen that in order to make the system regulation ability meet the threshold condition of insufficient supply and demand balance rate of less than $10 \%$, the optimal scheme has been modified and improved the regulation rate parameters of each conventional generator set, but the unit has not been expanded. This is because the power capacity 
resources of the system are relatively abundant and the sum of the theoretical output of conventional power generation resources and renewable energy resources far exceeds the load power supply requirements of the system. At the same time, even if there is an extreme situation where the output of renewable energy is zero, the upper limit of the capacity allocation of conventional power generation resources can meet the load power. The main contradiction that causes the supply and demand balance to remain unmaintained is that the system cannot compensate for the regulation demand brought about by changes in renewable energy power in a relatively short time scale. Therefore, the planning model optimizes the regulation capability parameters of the conventional generator set, so that the regulation capability of the system can be improved. It can follow the magnitude and rate of the response to the regulation demand and truly releases the capacity advantage of the system. Therefore, under the new background of renewable energy grid connection, the main demand for power system regulation has changed from capacity demand to demand for rapid regulation capability.

The objective value of optimal grid planning scheme in Table 1 is given in Table 2. As can be seen from the results that although the economically optimal scheme can guarantee various safety constraints operation and meet the power supply requirements of the load, its power system status and structural adaptability indexes are weaker than the comprehensive optimal scheme. This can be understood as that although the comprehensive optimal scheme sacrifices part of the economy, it effectively considers the operating state and adaptability of the structure in the planning process and has a more reasonable operating state and a more robust grid structure. It can better adapt to the development trend of large-scale grid connection of renewable energy. At the same time, the comprehensive optimal plan and the power supply construction and operation plan have a better matching degree. The following is a further analysis of the specific differences between the operating state and the network structure.

Table 2. Objective value for optimal grid planning scheme.

\begin{tabular}{ccc}
\hline Items & Comprehensive Optimal Plan & Economic Optimal Plan \\
\hline cost of grid expansion/Ten thousand yuan & $52,583.84$ & $34,668.75$ \\
joint weighted entropy index of operation state & 1.02 & 2.75 \\
joint weighted entropy index of network structure & 9493.70 & $10,668.53$ \\
\hline
\end{tabular}

\subsubsection{Comparison for Adaptability of Supply and Demand Balance}

The adaptability index of supply and demand balance is shown in Table 3. As we can see, the technical parameters have been improved, which has significantly improved the balance between supply and demand in the system. Before the transformation, the insufficient supply and demand balance rate of each typical scenario exceeded $20 \%$. In the scenario where the demand for renewable energy regulation is relatively frequent, this index reaches a maximum of $46.87 \%$. At the same time, the indexes of the upward and downward balance of supply and demand balance before the transformation are both large, indicating that there are frequent cases of urgent load or the curtailment of renewable energy. After the power supply adaptability evaluation and transformation, IRSD of each typical scenario are reduced to below $10 \%$. Further analysis shows that due to the threshold constraint, all scenarios need to meet the supply and demand balance shortage rate of less than $10 \%$. Therefore, the transformation result needs to ensure that the scene with the most stringent regulation requirements satisfies this condition and also makes the index far less than $10 \%$ in the remaining scenes, which is about $5 \%$. Secondly, IRUR decreased by an average of $86.09 \%$ compared with that before the transformation, indicating that the improvement of the unit's regulation capability reduced the urgent load phenomenon caused by the insufficient power up-regulation capability and improved the reliability of power supply. Similarly, IRDR decreased by an average of $80.99 \%$, indicating that the improvement of the unit's regulation capability reduced the phenomenon of renewable energy curtailment due to insufficient power down-regulation and increased the level of renewable energy consumption. 
Table 3. Comparison for adaptability index of supply and demand balance.

\begin{tabular}{ccccc}
\hline Items & Scenario & IRSD & IRUR & IRDR \\
\hline \multirow{3}{*}{ Before expansion } & 1 & $46.87 \%$ & $48.94 \%$ & $45.83 \%$ \\
& 2 & $30.21 \%$ & $36.17 \%$ & $25.00 \%$ \\
& 3 & $22.92 \%$ & $21.28 \%$ & $25.00 \%$ \\
After expansion & 4 & $46.87 \%$ & $56.25 \%$ & $38.30 \%$ \\
\hline & 2 & $9.37 \%$ & $4.26 \%$ & $14.58 \%$ \\
& 3 & $5.21 \%$ & $4.26 \%$ & $6.25 \%$ \\
& 4 & $6.12 \%$ & $4.26 \%$ & $2.08 \%$ \\
\hline
\end{tabular}

\subsubsection{Comparison for Adaptability of Operation State}

Table 4 shows the specific distribution of the expected load rate in the two optimal plans. The first column is the load rate interval, which is used to evaluate the reasonable degree of line load level. In this example, the rational ranking of each interval is set as: $(40-60 \%)>(20-40 \%)>(0-20 \%)>(60-80 \%)$ $>(80-100 \%)$. It can be seen from the results that the comprehensive optimal scheme does not have lines running in the last two intervals of the rational order of load levels and the economically optimal scheme has lines distributed in each interval and the penetration of lines above $80 \%$ load rate is as high as $19 \%$. It can be seen that the economic optimal plan has a situation where the load level of individual lines is too high and the concentration of its overall operating state is significantly weaker than the comprehensive optimal plan. From the self-organized critical theory, it can be seen that the safety level of the grid state of the economically optimal solution is poor. On the contrary, the comprehensive optimal scheme not only makes the grid state more orderly, but also ensures the efficiency of grid operation to the greatest extent. A total of $54 \%$ of the lines in the comprehensive optimal plan operate in the most reasonable load rate range preset by planners, while the data for the economically optimal plan is only $25 \%$. This shows that the running state of the comprehensive optimal scheme is more reasonable, taking into account the running efficiency and safety and is more in line with the running ideal state preset by the planning operator. Although the absolute value of efficiency is higher in the economically optimal solution, it is actually because some heavy-load lines increase the overall value and its true efficiency is significantly worse than the comprehensive optimal solution. It can be seen that the comprehensive optimal scheme has higher operating quality.

Table 4. Distribution of expected line load rate.

\begin{tabular}{ccccc}
\hline \multirow{2}{*}{ Interval of Load Rate } & \multicolumn{2}{c}{ Comprehensive Optimal Plan } & \multicolumn{2}{c}{ Economic Optimal Plan } \\
\cline { 2 - 5 } & Number of Lines & Penetration & Number of Lines & Penetration \\
\hline $0-20 \%$ & 11 & $27 \%$ & 10 & $31 \%$ \\
$20-40 \%$ & 8 & $20 \%$ & 3 & $9 \%$ \\
$40-60 \%$ & 22 & $54 \%$ & 8 & $25 \%$ \\
$60-80 \%$ & 0 & $0 \%$ & 5 & $16 \%$ \\
$80-100 \%$ & 0 & $0 \%$ & 6 & $19 \%$ \\
\hline
\end{tabular}

Table 5 shows the specific distribution of the power fluctuation rate of each line of the two schemes. Lines whose actual maximum load rate is less than $10 \%$ are not considered, because the absolute load level of such lines is too low and small changes in power will cause a large power fluctuation rate index, but the absolute magnitude of the change in load level is still small. It can be seen from the table that the fluctuation rate of $86 \%$ line in the comprehensive optimal scheme is below $20 \%$ of its allowable fluctuation interval, of which the line power fluctuation rate is less than $10 \%$ and the penetration of lines is $71 \%$. The two data corresponding to the optimal economic plan correspond to $71 \%$ and $21 \%$, respectively. In summary, the operating state of the optimal scheme is more stable, which can better resist the impact of renewable energy power fluctuations on the system state under uncertain 
operating environments and always maintain the safety and efficiency of the operating state during actual operation. At the same time, it can be found that the two schemes still have a small amount of line power fluctuation rate, which is because the line mainly undertakes the task of renewable energy transmission. Large fluctuations in the output of renewable energy have led to large fluctuations in the power of these lines, but they have not had a significant impact on the overall operating state.

Table 5. Distribution of line power fluctuation rate.

\begin{tabular}{ccccc}
\hline \multirow{2}{*}{ Interval of Fluctuation Rate } & \multicolumn{2}{c}{ Comprehensive Optimal Plan } & \multicolumn{2}{c}{ Economic Optimal Plan } \\
\cline { 2 - 5 } & Number of Lines & Penetration & Number of Lines & Penetration \\
\hline $0-10 \%$ & 24 & $71 \%$ & 6 & $21 \%$ \\
$10-20 \%$ & 5 & $15 \%$ & 14 & $50 \%$ \\
$20-30 \%$ & 3 & $9 \%$ & 6 & $21 \%$ \\
$30-40 \%$ & 0 & $0 \%$ & 0 & $0 \%$ \\
$40-50 \%$ & 2 & $6 \%$ & 1 & $4 \%$ \\
$50-60 \%$ & 0 & $0 \%$ & 0 & $4 \%$ \\
$60-70 \%$ & 0 & $0 \%$ & 1 & $0 \%$ \\
$70-80 \%$ & 0 & $0 \%$ & 0 & $0 \%$ \\
$80-90 \%$ & 0 & $0 \%$ & 0 & $0 \%$ \\
$90-100 \%$ & 0 & $0 \%$ & 0 & \\
\hline
\end{tabular}

\subsubsection{Comparison for Adaptability of Network Structure}

Tables 6 and 7 show the distribution of the interval of the electric betweenness of buses and branches of the two schemes. The first column in the table is the interval of the electric dielectric value, which is the objective standard used to evaluate the robustness degree of branch and bus. The higher the ratio of the number of components in the smaller area is, the more reasonable and robust network structure is. The results show that $88 \%$ of the total number of branches in the comprehensive optimal scheme are distributed in the range of (0-80) low electric medium and $81 \%$ in the economic optimal scheme. In the same way, $72 \%$ of the total buses of the comprehensive optimal scheme are distributed in the (0-80) low electric medium range, while the data of the economic optimal scheme are only $56 \%$. In conclusion, in the comprehensive optimal scheme, the electrical mediums of branches and buses are more centralized and orderly distributed in the range of low severity, the rationality of its network structure is obviously superior to the economic optimal scheme and its resistance and adaptability to uncertain impact are stronger.

Table 6. Distribution of electric betweenness of bus.

\begin{tabular}{ccccc}
\hline \multirow{2}{*}{ Interval of Electric Betweenness } & \multicolumn{2}{c}{ Comprehensive Optimal Plan } & \multicolumn{2}{c}{ Economic Optimal Plan } \\
\cline { 2 - 5 } & Number of Buses & Penetration & Number of Buses & Penetration \\
\hline $0-40$ & 7 & $39 \%$ & 7 & $39 \%$ \\
$40-80$ & 6 & $33 \%$ & 3 & $17 \%$ \\
$80-120$ & 1 & $6 \%$ & 4 & $22 \%$ \\
$120-160$ & 4 & $22 \%$ & 4 & $22 \%$ \\
$160-200$ & 0 & $0 \%$ & 0 & $0 \%$ \\
\hline
\end{tabular}


Table 7. Distribution of electric betweenness of branch.

\begin{tabular}{ccccc}
\hline \multirow{2}{*}{ Interval of Electric Betweenness } & \multicolumn{2}{c}{ Comprehensive Optimal Plan } & \multicolumn{2}{c}{ Economic Optimal Plan } \\
\cline { 2 - 5 } & Number of Lines & Penetration & Number of Lines & Penetration \\
\hline $0-40$ & 20 & $49 \%$ & 16 & $50 \%$ \\
$40-80$ & 16 & $39 \%$ & 10 & $31 \%$ \\
$80-120$ & 1 & $2 \%$ & 4 & $13 \%$ \\
$120-160$ & 4 & $10 \%$ & 2 & $6 \%$ \\
$160-200$ & 0 & $0 \%$ & 0 & $0 \%$ \\
\hline
\end{tabular}

\section{Conclusions}

From the perspective of adapting to the security integration of renewable energy, this paper firstly proposes the adaptability indexes system and applies it to the two-stage adaptable expansion planning of the transmission grid. The following conclusions can be draw:

(1) The adaptability of supply and demand balance not only evaluates the power balance of the system, but also focuses on the adequacy of the system regulation capacity and rate, which can reflect the level of renewable energy consumption and the risk of being urgently loaded; the adaptability of operation state consider the characteristics of power flow distribution change caused by renewable energy output fluctuation and the security, efficiency and stability of the actual operation of system; the adaptability of network structure evaluation the balance and robustness of system based on the order and severity of the electric betweennesses.

(2) The weighted entropy index can evaluate the adaptability state and network structure based on the given objective criteria. Not only can it achieve the ranking of relative advantages and disadvantages between the programs, but also it can refine the specific differences of program states and structures based on the objective standards. The objective evaluation criteria can be set by planner according to different considerations in different development periods, which improves the flexibility and applicability of the indexes. The application of joint weighted entropy can combine the physical meaning of each index to achieve dimensionality reduction and integration of indexes, so as to comprehensively measure the security of the state and structure with a single value. It is more suitable for the analysis safety adaptability under multi-dimensional influence factors and the multi-objective planning.

(3) The expansion planning of the high renewable energy penetrated system is vital and decisive for the actual consumption capacity in the operation stage, both the planning of the power plant in the source side and transmission line in the grid side are interrelated. For the improvement of safe integration of renewable energy in the planning stage, it is suggested to comprehensively consider the adaptability of supply and demand balance, operation state, and network structure in source-grid planning issues.

Author Contributions: Conceptualization, M.T.; methodology, J.W.; validation, J.W. and X.W.; writing一original draft preparation, J.W.; writing-review and editing, M.T. and X.W. All authors have read and agreed to the published version of the manuscript.

Funding: This work is founded by the National Key R\&D Program of China (2018YFB0905200)—Research and application demonstration on complementary combined power generation technology for distributed photovoltaic and cascade hydropower.

Conflicts of Interest: The authors declare no conflict of interest. 


\section{Nomenclature}

\begin{tabular}{|c|c|}
\hline$P_{\mathrm{D}}^{\text {up }}, P_{\mathrm{D}}^{\text {down }}$ & up- and down-regulation demand (MW) \\
\hline$P_{i, \mathrm{REG}}$ & output power of the renewable energy unit $i(\mathrm{MW})$ \\
\hline$\Omega_{\text {REG }}$ & the set for renewable energy units \\
\hline$P_{\mathrm{S}}{ }^{\text {up }}, P_{\mathrm{S}}$ down & up- and down-regulation capability (MW) \\
\hline$\Omega_{\mathrm{G}}$ & the set of fast regulating units \\
\hline$P_{i, \mathrm{G}}$ & output power of the fast regulating units $i$ at time $t(\mathrm{MW})$ \\
\hline$P_{i, \mathrm{G}}^{\max }, P_{i, \mathrm{G}}^{\min }$ & upper and lower output of the fast regulating generator units (MW) \\
\hline $\mathrm{R}_{\mathrm{i}, \mathrm{g}}$ up, $\mathrm{R}_{\mathrm{i}, \mathrm{g}}$ down & maximum up and down ramp rate of the fast regulation units (MW/15min) \\
\hline $\mathrm{P}_{\mathrm{M}}^{\text {up }, \mathrm{P}_{\mathrm{M}} \text { down }}$ & upper and lower regulation resource margins (MW) \\
\hline$T_{\text {ins }}$ up & the time when the up-regulation resources are insufficient \\
\hline$T_{\text {total }}^{\text {up }}$ & the total operation time when the up-regulation demand occurs \\
\hline$T_{\text {ins }}$ down & the time when the down-regulation resource is insufficient \\
\hline$T_{\text {total }}$ down & the total operation time when the down-regulation demand occurs \\
\hline$P_{i}$ & the actual transmission power of line $i$ \\
\hline$S_{i, \max }$ & the rated transmission power capacity of the line \\
\hline$\omega_{\mathrm{a}}, \omega_{\mathrm{b}}$ & the weights of generator and load bus \\
\hline$B_{e, a b}(i)$ & the current distribution of bus $i$ \\
\hline$I_{a b}(i, j)$ & the current of branches $(i, j)$ \\
\hline$C_{\mathrm{G}}$ & the cost of regulation capacity transformation \\
\hline$C_{W R}$ & the annual cost of renewable energy generation \\
\hline$C_{W L}$ & the annual load shedding cost \\
\hline$C_{\mathrm{N}}$ & the cost of grid expansion \\
\hline$\Sigma P_{\mathrm{L}}(s, t)$ & the total load at time slot $t$ in scenario $s$ \\
\hline IRSD & insufficient rate of supply and demand balance \\
\hline IRUR & insufficient rate of up-regulation \\
\hline IRDR & insufficient rate of down-regulation \\
\hline AIUR & average insufficiency of up-regulation \\
\hline AIDR & average insufficiency of down-regulation \\
\hline WEELLR & weighted entropy of expected line load rate \\
\hline WELPFR & weighted entropy of line power fluctuation rate \\
\hline JWEOS & joint weighted entropy of operation state \\
\hline WEEBBus & weighted entropy for electric betweenness of bus \\
\hline WEEBBra & weighted entropy for electric betweenness of branch \\
\hline JWENS & Joint weighted entropy of network structure \\
\hline
\end{tabular}

\section{References}

1. Eitan, A.; Rosen, G.; Herman, L.; Fishhendler, I. Renewable energy entrepreneurs: A conceptual framework. Energies 2020, 13, 2554. [CrossRef]

2. Murdock, H.E.; Gibb, D.; André, T. Renewables 2019 Global Status Report; REN21: Paris, France, 2019.

3. Kiviluoma, J.; Holttinen, H.; Weir, D.; Scharff, R.; Soder, L.; Menemenlis, N.; Cutululis, N.A.; Danti Lopez, I.; Lannoye, E.; Estanqueiro, A.; et al. Variability in large-scale wind power generation. Wind Energy 2016, 19, 1649-1665. [CrossRef]

4. Zsiborács, H.; Baranyai, N.H.; Vincze, A.; Zentkó, L.; Birkner, Z.; Máté, K.; Pintér, G. Intermittent Renewable Energy Sources: The Role of Energy Storage in the European Power System of 2040. Electronics 2019, 8, 729. [CrossRef]

5. Lu, Z.; Huang, H.; Shan, B.; Wang, Y.; Du, S.; Li, J. Morphological evolution model and power forecasting prospect of future electric power systems with high proportion of renewable energy. Autom. Electr. Power Syst. 2017, 41, 12-18.

6. Lannoye, E.; Flynn, D.; O'Malley, M. Transmission, variable generation, and power system flexibility. IEEE Trans. Power Syst. 2015, 30, 57-66. [CrossRef]

7. Wheelan, S.A. Harnessing Variable Renewables; International Energy Agency: Paris, France, 2011. 
8. Lannoye, E.; Flynn, D.; O’Malley, M. Evaluation flexibility. IEEE Trans. Power Syst. 2012, 27, $922-931$. [CrossRef]

9. Nosair, H.; Bouffard, F. Flexibility envelopes for power system operational planning. IEEE Trans. Sustain. Energ. 2015, 6, 800-809. [CrossRef]

10. Ma, J.; Silva, V.; Belhomme, R.; Kirschen, D.S.; Ochoa, L.F. Evaluating and planning flexibility in sustainable power systems. IEEE Trans. Sustain. Energ. 2013, 4, 200-209. [CrossRef]

11. Zhuo, Z.; Du, E.; Zhang, N.; Kang, C.; Xia, Q.; Wang, Z. Incorporating Massive Scenarios in Transmission Expansion Planning with High Renewable Energy Penetration. IEEE Trans. Power Syst. 2020, 35, 1061-1074. [CrossRef]

12. Li, X.; Fan, J.; Cao, L.; Wang, L.; Hu, T.; Wu, T.; Zhou, Z. Analysis on the adaptability of large-scale grid-connected PV station. Power Sys. Prot. Control 2018, 46, 164-169.

13. Liu, W.; Cai, W.; Yangqing, D.; Liang, C.; Wang, J.; Wang, N.; Ma, Y. The evolution of grid's self-organized criticality state based on entropy theory under the circumstance of large-scale wind power centralized grid. Power Syst. Technol. 2013, 37, 3392-3398.

14. Zhang, C.; Cheng, H.; Liu, L.; Wang, Z.; Lu, J.; Zhang, X. Adaptability index and evaluation method for power transmission network structure with integration of high penetration of renewable energy. Autom. Electr. Power Syst. 2017, 41, 55-61.

15. Dai, W.; Yang, Z.; Yu, J.; Zhao, K.; Wen, S.; Lin, W.; Li, W. Security region of renewable energy integration: Characterization and flexibility. Energy 2019, 187, 115975. [CrossRef]

16. Papavasiliou, A.; Oren, S.S. Large-Scale integration of deferrable demand and renewable energy sources. IEEE Trans. Power Syst. 2014, 29, 489-499. [CrossRef]

17. Khan, S.U.; Mehmood, K.K.; Haider, Z.M.; Rafique, M.K.; Kim, C. A bi-level EV aggregator coordination scheme for load variance minimization with renewable energy penetration adaptability. Energies 2018, 11, 2809. [CrossRef]

18. Sun, W.; Wang, C.; Zeng, P.; Han, J. Review on evaluation method and index of power system homogeneity. Power Syst. Technol. 2015, 39, 1205-1212.

19. Liu, W.; Cai, W.; Zhang, N.; Dan, Y.; Liang, C.; Wang, W.; Liang, C. Evolution of self-organizing of grid critical state based on united weighted entropy theory. Proc. CSEE 2015, 35, 1363-1370.

20. Sheng, S.; Gu, Q. A day-ahead and day-in decision model considering the uncertainty of multiple kinds of demand response. Energies 2019, 12, 1711. [CrossRef]

21. Ji, W.; Wu, J.; Zhang, M.; Liu, Z.; Shi, G.; Xie, X. Blind image quality assessment with joint entropy degradation. IEEE Access 2019, 7, 30925-30936. [CrossRef]

22. Bai, H.; Miao, S. Hybrid flow betweenness approach for identification of vulnerable line in power system. IET Gener. Transm. Distrib. 2015, 9, 1324-1331. [CrossRef]

23. Teimourzadeh, S.; Aminifar, F. MILP Formulation for Transmission Expansion Planning with Short-Circuit Level Constraints. IEEE Trans. Power Syst. 2016, 31, 3109-3118. [CrossRef]

24. Cheng, Y.; Zhang, N.; Wang, J.; Li, H.; Wang, Z.; Xie, L.; Kang, C. Comprehensive Evaluation of Transmission Network Planning for Integration of High-penetration Renewable Energy. Autom. Electr. Power Syst. 2019, $43,33-42 ; 57$.

25. Huang, Y.; Li, Y.; Gao, C. Multi-objective transmission network planning based on non-dominated sorting differential evolution. Dianwang Jishu/Power Syst. Technol. 2011, 35, 85-89.

(C) 2020 by the authors. Licensee MDPI, Basel, Switzerland. This article is an open access article distributed under the terms and conditions of the Creative Commons Attribution (CC BY) license (http://creativecommons.org/licenses/by/4.0/). 